\title{
Asociación entre la presencia de anticuerpos anti-Ras y anti-VPH16 E 4/E 7 y lesiones intraepiteliales del cérvix
}

SaraVázquez-Corzo, Lic en Q FB, M en C, ${ }^{(1-3)}$ Catalina Trejo-Becerril, Biól, M en C, ${ }^{(2)}$ A urelio Cruz-Valdez, MC, MSP, ${ }^{(3)}$ Pilar Hernández-N evarez, Psic, ${ }^{(3)}$ Fernando Roger Esquivel-Guadarrama, Biól, PhD, ${ }^{(4)}$

Ma de Lourdes Gutiérrez-X icotencatl, Biól, PhD, ${ }^{(1)}$ Grupo de trabajo de Cáncer Cérvico Uterino*

Vázquez-Corzo S,Trejo-Becerril C, Cruz-Valdez A, Hernández-Nevarez P, Esquivel-Guadarrama FR, Gutiérrez-Xicotencatl ML.

Grupo de trabajo de Cáncer Cérvico Uterino. Asociación entre la presencia de anticuerpos anti-Ras y anti-VPH16 E4/E7 y lesiones intraepiteliales del cérvix. Salud Publica Mex 2003;45:335-345.

El texto completo en inglés de este artículo está disponible en: http://www.insp.mx/salud/index.html

\section{Resumen}

Objetivo. D eterminar si anticuer pos séricos contra E4, E7 y Ras pueden ser utilizados como marcadores de lesiones tempranas del cérvix uterino asociadas al virus del papiloma humano. Material y métodos. Entre marzo de 1999 y abril de 2000 se realizó un estudio sero-epidemiológico de casos y controles en la clínica de displasias del Hospital General Doctor Gea González, en la Ciudad de Mexico, en 116 muestras de suero para evaluar la presencia de anticuerpos anti-E4, E7 y Ras utilizando un ELISA de captura. Se estimaron razones de momios e intervalos de confianza de $95 \%$ Resultados. Anticuerpos anti-E7 se asociaron a mujeres con lesiones N IC III, mientras que anticuerpos antiE4 y anti-Ras fueron más frecuentes en lesiones N IC I-II. AI evaluar el perfil de anticuerpos que presentar on las mujeres, encontramos que a) anticuerpos contra dos proteínas predicen la existencia de una lesion N IC I-II, y b) la presencia de

\author{
Vázquez-Corzo S,Trejo-Becerril C, Cruz-Valdez A, \\ Hernández-Nevarez P, Esquivel-Guadarrama FR, \\ Gutiérrez-Xicotencatl ML. \\ Grupo de trabajo de Cáncer Cérvico Uterino. \\ Association between anti-Ras and \\ anti-HPV16E4/E7 antibodies with cervical intraepithelial lesions. \\ Salud Publica Mex 2003;45:335-345. \\ The English version of this paper \\ is available at: http://www.insp. mx/salud/index.html
}

\begin{abstract}
A bstract
Objective. To evaluate whether serum antibodies anti-E4, E7 and Ras could be used as markers for early cervical lesions associated with H PV (human papillomavirus). Material and Methods. A seroepidemiological case-control study was conducted between March 1999 and April 2000 at the dysplasia clinic of Hospital G eneral D octor G ea Gonzalez, in Mexico City, to evaluate the presence of antibodies antiE4, E7, and Ras through a sandwich ELISA. Analysis was done using odds ratios and $95 \%$ confidence intervals. Results.Anti-E7 antibodies were associated to women with CIN III lesions, while anti-E4 and Ras antibodies were strongly associated with CIN I-II lesions. The antibody profile of women with different cervical lesion showed that: a) the presence of antibodies against two proteins predicts the presence of CIN I-II lesions, and b) the presence of three antibodies predicts a CIN III lesion.
\end{abstract}

Este trabajo fue apoyado por los financiamientos del Instituto N acional de Salud Pública (Proyecto N o. 203) y Fundación "Miguel Alemán" AC. A simismo, fue presentado en el 110 Congreso Internacional de Inmunología, en Estocolmo, Suecia, julio de 2001.

(1) Centro de Investigación sobre Enfermedades Infecciosas, Instituto N acional de Salud Pública (IN SP), Cuernavaca, Morelos, México.

(2) Departamento de Investigación Básica, Instituto N acional de Cancerología, México, DF, México.

(3) Centro de Investigación en Salud Poblacional, IN SP, Cuernavaca, Morelos, México.

(4) Facultad de Medicina, Universidad Autónoma del Estado de Morelos, Cuernavaca, Morelos, México.

(5) Clínica de Displasias, Hospital General D octor Gea González, México, D F, México.

* Grupo de trabajo de Cáncer Cérvico Uterino:Adolfo Pedroza-Saavedra, IQ ,M en C, ${ }^{(1)}$ Minerva Maldonado-G ama, Biól,(1) Humberto Valdovinos-Torres, Biól, (1) Leopoldo Vázquez-Estrada, MC (5)

Fecha de recibido: 8 de octubre de 2002 - Fecha de aprobado: 23 de abril de 2003

Solicitud de sobretiros: D ra. Ma de Lourdes Gutiérrez X icotencatl.A venida U niversidad 655, colonia Santa María A huacatitlán 62508, Cuernavaca, Morelos, México.

Correo electrónico:mlxico@ correo.insp.mx 
tres anticuerpos predicen una lesión N IC III. Conclusiones. La detección de anticuerpos séricos contra E4, E7 y Ras en combinación con otras técnicas de diagnóstico, podrían ser de utilidad para detectar oportunamente a mujeres con lesiones tempranas aso ciadas alVirus del Papilo ma H umano y en riesgo de desarrollar cáncer. El texto completo en inglés de este artículo está disponible en: http://www.insp.mx/salud/index.html

Palabras clave: papilo mavirus humano; anticuerpos séricos; lesiones tempranas de cérvix uterino; antígenos virales; oncogén Ras; México
Conclusions. The presence of serum antibodies against E4, E7 and Ras, to gether with other diagnostic techniques, could be useful for the timely detection of early uterine cervical lesions associated to HPV in women at risk of developing cancer.The English version of this paper is available at: http:/ /www.insp.mx/salud/index.html

Key words: papillomavirus, human; serum antibodies; early lesions of the uterine cervix; antigens, viral; Ras oncogene; Mexico
E 1 cáncer cérvico uterino $(\mathrm{CaCu})$ continúa siendo uno de los principales problemas de salud en países en desarrollo. ${ }^{1}$ En México el $\mathrm{CaCu}$ es la primera causa de muerte por neoplasias y representa $35 \%$ de los tumores malignos en la mujer. ${ }^{2}$

Diversas investigaciones epidemiológicas han demostrado que los Virus del Papiloma Humano (VPH) son el factor etiológico más importante en el desarrollo del $\mathrm{CaCu}_{1}{ }^{3}$ y de éstos los tipos 16 y 18, considerados de alto riesgo por su asociación con neoplasias anogenitales, son los más frecuentes. ${ }^{1}$ El genoma del VPH contiene una región de expresión temprana, una de expresión tardía y una región larga de control, la cual contiene el origen de replicación y transcripción viral. La región temprana codifica para proteínas relacionadas con replicación (E1), activación o represión (E2) del DNA viral, producción de partículas virales (E4) y de transformación celular (E5, E6 y E7), mientras que la región tardía codifica para proteínas de la cápside (L1 y L2). ${ }^{1}$ Se ha identificado que los genes E6 y E7 de los VPH de alto riesgo están presentes en la mayoría de los carcinomas cervicales y en líneas celulares derivadas de éstos, y son los principales causantes del desarrollo tumoral debido a su capacidad oncogénica. ${ }^{4}$ Por otra parte, la expresión de la proteína E7 del VPH16 o 18 en cooperación con la proteína celular Ras es suficiente para transformar cultivos de células primarias de ratón. ${ }^{5,6}$ Una de las proteínas del VPH que se ha identificado en los estadios tempranos de la infección es la proteína E4. Se ha demostrado que E4 se expresa tempranamente en la capa parabasal del epitelio cervical, asociada a la replicación vegetativa del DNA viral, manteniendo su expresión a lo largo de varias capas celulares. Esta expresión se correlaciona con una disminución de la expresión de queratinas, lo que explicaría la ausencia de queratinas de diferenciación terminal en queratinocitos. Asimismo, se ha observado que E4 del VPH16 puede causar el colapso de los filamentos de citoqueratina y afectar la orga- nización de la membrana plasmática, las señales de transducción y el crecimiento celular. ${ }^{7,8}$

La respuesta inmune humoral en la infección por el VPH no ha sido estudiada de manera sistemática, debido a que la respuesta de anticuerpos no juega un papel importante en la eliminación de esta infección. Sin embargo, ha servido como marcador de estadios avanzados del $\mathrm{CaCu}$, con base en la presencia de anticuerpos anti-E7 de VPH16 que se han asociado a cáncer epidermoide. ${ }^{9,10}$ De la misma manera, la presencia de anticuerpos anti-E6 del VPH16 puede ser un marcador para cáncer invasor. ${ }^{11}$ En ensayos de ELISA se han detectado anticuerpos contra L1 y L2 tanto en pacientes como en personas sanas ${ }^{12,13} \mathrm{y}$, por analogía con otras infecciones virales, sugieren una infección previa, mientras que la presencia de anticuerpos contra proteínas tempranas pueden reflejar una infección reciente. ${ }^{14}$ Actualmente, son limitados y contradictorios los reportes en relación con la presencia de anticuerpos contra E4, en los cuales algunos investigadores han reportado que anticuerpos anti-E4 del VPH16 presentaron prevalencias hasta de $40 \%$ en pacientes con diferentes grados de lesión cervical, en cánceres no anogenitales, en pacientes inmunosuprimidos y en mujeres sanas. ${ }^{9,15}$ Otros investigadores identificaron que anticuerpos anti-E4 se presentaron con mayor prevalencia en mujeres con cáncer que en el grupo control. ${ }^{1,16}$ Estudios previos en nuestro laboratorio mostraron que anticuerpos contra E4 de VPH16 se encuentran en baja proporción en mujeres sanas (9\%), lo cual se eleva hasta en $65 \%$ en lesiones del tipo NICI-III y en pacientes con cáncer cervical, lo que sugiere que éstos pudieran servir como marcadores tempranos de la enfermedad. ${ }^{17}$

Dentro de los factores que contribuyen a la progresión de las lesiones de bajo a alto grado están la generación de una respuesta inmune deficiente, coinfecciones, mutágenos y hormonas, entre otros. ${ }^{18}$ También se ha determinado qué factores celulares pueden estar involucrados en el desarrollo del $\mathrm{CaCu}$, debido a 
alteraciones en proto-oncogenes ( $m y c$, ras) y anti-oncogenes (p53, Rb). ${ }^{19-21}$ En CaCu se ha reportado que la sobrexpresión de la proteína Ras parece tener cierta importancia en el desarrollo de lesiones tempranas (28\%) y se incrementa hasta en $54 \%$ conforme la lesión avanza hacia NIC III y carcinoma microinvasivo. ${ }^{19}$

Actualmente el diagnóstico temprano para identificar una lesión o una infección por el VPH es todavía difícil, ya que sistemas de detección como la citología y la colposcopía que localizan lesiones en cérvix asociadas al VPH, lo hacen cuando éstas están avanzadas. Recientemente se han desarrollado técnicas como el hybrid capture y el sistema de reverse line-blot detection method, ${ }^{22,23}$ las cuales han sido utilizadas como sistemas más sensibles en la detección de secuencias de DNA del VPH. Sin embargo, la desventaja de estos sistemas es que no se puede determinar si la infección por el VPH en los individuos positivos a éste se refiere a una infección activa o a un virus latente, que en el caso del papiloma el proceso, desde la infección inicial hasta el desarrollo del cáncer, puede llevarse hasta en un periodo de 20 años. ${ }^{24}$ Por ello, pensamos que el análisis de la respuesta de anticuerpos séricos contra proteínas tempranas del VPH será de gran importancia para evaluar la progresión de la lesión generada por la infección por este virus, ya que la generación de anticuerpos contra proteínas del virus nos darán una medida indirecta de la actividad del papilomavirus. Nuestro grupo demostró previamente la asociación entre anticuerpos anti-E4 y Ras con las lesiones NIC y la presencia de DNA del VPH16. Sin embargo, no pudimos definir el estadio específico al que estos anticuerpos se asociaban, debido al bajo número de muestras en este grupo. Por lo que, en este estudio propusimos evaluar individualmente y en combinación, la utilidad de medir anticuerpos anti-E4, E7 y Ras como posibles marcadores de lesiones tempranas del cérvix uterino (NIC I, II y III), utilizando para esto un ensayo de ELISA de captura.

\section{Material y métodos}

\section{Población de estudio}

Se realizó un estudio de casos y controles, entre marzo de 1999 y abril de 2000. En total se analizaron 116 muestras de mujeres, de las cuales 43 fueron casos atendidos en la Clínica de Displasias del Hospital General Doctor Gea González de la Ciudad de México, DF, de entre 25 a 60 años de edad $(\bar{X}=36 ; D S=8.04)$ y 73 controles de 25 a 65 años $(\bar{X}=35 ; D S=7.7)$ seleccionados aleatoriamente por estratos de edad de un estudio poblacional realizado en el estado de Morelos. La se- mejanza de las poblaciones en estudio se evaluó de acuerdo con las diferencias estadísticas de factores predictores de $\mathrm{CaCu}$ (edad, antecedentes reproductivos, conducta sexual y antecedentes de infecciones de transmisión sexual), utilizando una base de datos de un estudio realizado en la Ciudad de México, ${ }^{25}$ y comparado con la población del estado de Morelos. ${ }^{26} \mathrm{Ambos}$ estudios fueron realizados con base poblacional de mujeres sin lesión cervical, en donde no se observaron diferencias estadísticamente significativas entre los factores de riesgo evaluados (datos no mostrados).

Posterior a la lectura y firma del consentimiento informado, todas las mujeres que aceptaron participar en el estudio, incluidas como casos, fueron confirmadas por examen colposcópico y por biopsia directa. Un total de 43 muestras de biopsias y sueros fueron colectadas de mujeres diagnosticadas con diferentes grados de alteración del cérvix uterino como cervicitis crónica e inflamación ( $n=11$, las cuales serán referidas como lesiones no neoplásicas en lo sucesivo), neoplasia intraepitelial cervical (NIC) grado I y II ( $n=12)$, y NIC III $(n=20)$. Un total de 73 muestras de sueros y raspados cervicales fueron seleccionadas como controles del banco de muestras del estudio del estado de Morelos, provenientes de mujeres con diagnóstico normal por estudio citológico, ${ }^{26}$ las cuales fueron pareadas por edad con los casos ( \pm 2 años).

\section{Detección y tipificación del VPH por reverse-line blot}

La determinación de los tipos del VPH fue estandarizada en el laboratorio del doctor K Shah, en el Departamento de Microbiología Molecular e Inmunología en Baltimore, MD, Estados Unidos de América (EUA) y las muestras del estudio procesadas en el Instituto Nacional de Salud Pública (INSP) en Cuernavaca, Morelos, México. El ensayo para el VPH utilizado fue el reverse line blot descrito por Gravitt y colaboradores. ${ }^{22} \mathrm{Un}$ fragmento de DNA de VPH fue amplificado utilizando los oligonucleótidos consenso de L1 biotinilados MYB09 5'-CGTCCMARR GGAWACTGATC-3' y el anti-sentido MYB11 5'-GCMCAGGGWCA TAAYAATGG- $3^{\prime}{ }^{27}$ Para determinar la calidad de las muestras se co-amplificó un fragmento del gen de $\beta$-globina con los oligonucleótidos GH20 5'-GAAGAGCCAAGG ACAGGTAC-3', y el anti-sentido BPCO4 5'-CAACTTCATCCACG TTCACC-3'. Para la tipificación del $\mathrm{VPH}$, los productos de PCR fueron hibridados a sondas inmovilizadas sobre soportes de plástico, de acuerdo con el protocolo de Roche System Mol. Inc. (Alameda, CA, EUA). Cada tira contenía los tipos de los VPH asociados con cáncer, los cuales fue- 
ron $16,18,26,31,33,35,39,45,51,52,53,56,58,59,66$, 88 y MM9. Asimismo, las tiras incluían los VPH no asociados con cáncer, los cuales fueron los tipos 6, 11, 40, 42, 54, 55, 57, MM4, MM7 y MM8. Las señales de hibridación fueron detectadas, por desarrollo de color, con peroxidasa de rábano y tetrametil bencidina, y la interpretación de los resultados se hizo de acuerdo con un patrón de bandas prestablecido. ${ }^{22}$

\section{Material biológico}

La proteína recombinante cII-Ras se obtuvo a partir del plásmido pJL6 que contenía el proto-oncogén cHa-ras, donado por el doctor J Lauthenberger (Lab. of Leukocyte Biology, NCI-FCRDC, Frederick, MD, EUA). La proteína GST-E4 fue obtenida del plásmido pGEX que contenía el gen E4 de VPH16, donado por el doctor J Doorbar (Nat Inst for Medical Res, Londres, Inglaterra) y la proteína GST-E7 fue obtenida del plásmido pGEX que contenía el gen E7 de VPH16, donado por el doctor E Lam (Imperial College School of Medicine, Hammersmith Hospital, Londres, Inglaterra).

\section{Producción de anticuerpos policlonales anti-GST y anti-Ras}

Los anticuerpos policlonales de conejo anti-Ras (C-138) y anti-GST (C357) fueron producidos en el Laboratorio de Virus y Cáncer del INSP. Brevemente, conejos de la cepa Nueva Zelanda fueron inmunizados intramuscularmente con $150 \mu \mathrm{g}$ de las proteínas recombinantes y emulsificadas por una ocasión con adyuvante completo de Freund (Gibco, Gaithersburg, MD, EUA). Posteriormente, se realizaron cuatro inmunizaciones más, en intervalos de 15 días, utilizando adyuvante incompleto de Freud. Los sueros de conejos inmunizados fueron seleccionados por medio de un ensayo de inmunoabsorvencia ligado a enzimas (enzyme-linked immunoabsorbent assay -ELISA-) utilizando proteínas recombinantes aisladas, como se menciona adelante.

\section{Expresión y aislamiento de proteínas recombinantes en bacterias}

Las proteínas recombinantes cII-Ras, GST-E4 y GSTE7 fueron inducidas por choque térmico a $42{ }^{\circ} \mathrm{C}$, la primera, y por inducción con $1 \mathrm{mM}$ de IPTG (Isopropil-tiogalacto-piranósido) para las proteínas fusionadas a GST. ${ }^{7,28}$ Las proteínas recombinantes así inducidas fueron aisladas de acuerdo con lo reportado por Pedroza-Saavedra y colaboradores. ${ }^{17}$ Brevemente, las bacterias se lisaron por $30 \mathrm{~min}$ a $4{ }^{\circ} \mathrm{C}$ en buffer TE $[50 \mathrm{mM}$ Tris- $\mathrm{HCl}, 2 \mathrm{mM}$ EDTA, $\mathrm{pH}$ 8.0, 2mM fluoruro de fe- nilmetil sulfonilo (PMSF), $0.2 \mathrm{mg} / \mathrm{ml}$ lisozima y $0.15 \%$ (v/v) Tritón X-100]. Los lisados se sonicaron con cinco pulsos de $20 \mathrm{seg}$ a $4{ }^{\circ} \mathrm{C}$ (Sonicador Brandson, VWR, EUA) y se centrifugaron a $8000 \mathrm{xg}$, por 30 minutos. Finalmente, el botón de proteínas obtenido por centrifugación, en donde se encontraron las proteínas recombinantes insolubles, se disolvió en $6 \mathrm{M}$ de urea, y se cuantificó la concentración de proteínas por medio del sistema de BCA de Pierce Chemical Company (Illi, EUA).

\section{Ensayo de ELISA de captura}

El ensayo de captura se llevó a cabo de acuerdo con lo descrito por Meschede y colaboradores. ${ }^{29}$ En general, la placa de ELISA se incubó a $4{ }^{\circ} \mathrm{C}$, por $16 \mathrm{~h}$, con el anticuerpo de captura (anti-Ras C168 o anti-GST C357) en buffer de carbonatos (18mM Na $\mathrm{CO}_{3}, 32 \mathrm{mM} \mathrm{NaH}-$ $\mathrm{CO}_{3} \mathrm{pH}$ 9.3). Después de cada incubación las placas se lavaron con PBS/Tween $\left(2.6 \mathrm{mM} \mathrm{KCl}, 1.5 \mathrm{mM} \mathrm{KH}_{2} \mathrm{PO}_{4^{\prime}}\right.$ $140 \mathrm{mM} \mathrm{NaCl}, 8.2 \mathrm{mM} \mathrm{NaH}_{2} \mathrm{PO}_{4^{\prime}}$ pH 7.0 y $0.05 \%$ de Tween 20). La placa se bloqueó con PBS/1\% BSA (albúmina sérica bovina, Sigma fracción V) por 2 h, a 37 ${ }^{\circ} \mathrm{C}$. Los antígenos (cII-Ras, GST-E4 o GST-E7) fueron diluidos en PBS y colocados a una concentración de $300 \mu \mathrm{g}$ por pozo, y se incubaron por $2 \mathrm{~h}$ a $37^{\circ} \mathrm{C}$. Los sueros problemas se colocaron por duplicado en diluciones de 1:50 y 1:100 en PBS/1\% BSA, y se incubaron por $16 \mathrm{~h}$ a $4{ }^{\circ} \mathrm{C}$. Finalmente, se adicionó el anticuerpo anti-inmunoglobulinas humanas (IgG, IgM e IgA) conjugado con peroxidasa (Sigma, EUA). La placa se reveló, a temperatura ambiente, con buffer de fosfatos-citrato (50mM Na $2 \mathrm{HPO}_{4}, 24 \mathrm{mM}$ ácido cítrico), que contenía $0.04 \%$ de orto-fenilendiamina y $0.012 \%$ de $\mathrm{H}_{2} \mathrm{O}_{2}$. La reacción fue detenida con $2 \mathrm{M} \mathrm{H}_{2} \mathrm{SO}_{4}$, y la placa leída a $492 \mathrm{~nm}$ en un lector de microplacas (Labsystem, EUA). Debido a que el fondo del sistema fue alto, aun con los sueros controles, se decidió manejar un valor de proporción, lo cual nos permitió tener una mejor separación de sueros positivos y negativos. El valor de proporción se obtuvo de la siguiente manera:

$\left[\mathrm{A}_{492 \mathrm{~nm}}\right.$ de pozo con proteína recombinante (E4, E7 o Ras) $/ \mathrm{A}_{492 \mathrm{~nm}}$ de pozo sin antígeno]

Los controles que se manejaron todo el tiempo en los ensayos y por placa de ELISA fueron: a) el control de reactivos el cual no llevaba suero problema, y b) el control sin antígeno para identificar posibles reacciones cruzadas del suero de captura con el suero problema. El valor de corte para cada uno de los anticuerpos se obtuvo de los valores de proporción (definido arriba) de 44 mujeres sin lesión cervical, con PAP normal y negativas para DNA del VPH. La determinación del 
valor de corte para inmunoglobulinas totales se llevó a cabo utilizando el valor proporción en la prueba estadística U de Mann-Whitney para variables no paramétricas. De esta manera, el valor de corte se tomó como la media más dos desviaciones estándar $(D S)$, resultando así un valor de corte para anti-E7 de 3.5, anti-E4 de 3.9 y para anti-Ras de 4.5 .

\section{A nálisis estadístico}

Se calcularon estadísticas univariadas para todas las variables. Las variables medidas sobre una escala continua fueron exploradas en términos de su distribución original y en categorías. Para evaluar la asociación entre los anticuerpos contra Ras, anti-E4 y anti-E7 y los factores de riesgo se calculó la razón de momios (RM) con intervalos de confianza de 95\%. Para evaluar el estimador verdadero se utilizó un modelo de regresión logística condicionado por edad, y ajustadas por variables que predicen la presencia de anticuerpos. Se crearon tres modelos de acuerdo con la variable dependiente de interés (anti-E4, anti-E7 y anti-Ras). Para evaluar la diferencia de medias entre las variables continuas se empleó el estadístico t-test, tomando como la existencia de diferencia estadísticamente significativa una $p<0.05$.

\section{Resultados}

\section{Prevalencia del VPH en la población de estudio}

Un total de 116 muestras de DNA fueron amplificadas y probadas con 27 diferentes sondas para la presencia del VPH en el ensayo de reverse-line blot. En el cuadro I se muestran las prevalencias de DNA del VPH en la población de estudio. En general, se puede observar que 31\% (36/116) de la población en estudio presentó algún tipo de VPH. En el caso de los VPH oncogénicos, éstos tuvieron una mayor prevalencia entre las mujeres que presentaron lesiones tipo NIC I-II (50\%) y NIC III (40\%) que entre las mujeres con lesiones no neoplásicas (18\%) y las mujeres con citología normal (23\%). Los VPH no oncogénicos tuvieron una prevalencia de 3\% (3/116), estando presentes en las lesiones tempranas. Al realizar un análisis estadístico de estos resultados se observó que la posibilidad de tener lesión del cérvix uterino se incrementa hasta 3.2 veces más cuando se tiene algún tipo del VPH (IC 95\% 1.35-7.5), lo cual fue estadísticamente significativo $(p<0.05)$ y confirma la asociación del VPH con el desarrollo del cáncer cervical encontrado en otros estudios. ${ }^{3}$

\section{Prevalencias de anticuerpos contra Ras y proteínas tempranas del VPH16 en sueros de pacientes con lesiones del cérvix uterino}

El ELISA de captura se llevó a cabo para todos los sueros con las proteínas específicas, y los resultados del análisis estadístico de estos ensayos se encuentran resumidos en el cuadro II. Los resultados mostraron que el grupo de los casos $(n=43)$ presentó una prevalencia de $60 \%(26 / 43, \mathrm{p}=0.002)$ para anticuerpos anti-E7, de $56 \%(24 / 43, p=0.1)$ para anticuerpos anti-E4 y de $51 \%$ $(22 / 43, p=0.002)$ para anticuerpos anti-Ras. Al separar el grupo de los casos por grados de lesión se identificó una prevalencia de hasta $70 \%(14 / 20)$ contra anti-

Cuadro I

Prevalencias de los VPh respecto al tipo de lesión en la población de estudio. MÉxıCO, 1999-2000

\begin{tabular}{|c|c|c|c|c|c|c|}
\hline Tipo de lesión & $\mathrm{N}$ total & $\begin{array}{l}\text { VPH } 16 \\
\text { Positivos }\end{array}$ & $\begin{array}{l}\text { VPH oncogénicos } \\
\text { No. }(\%)\end{array}$ & $\begin{array}{c}\text { VPH no oncogénicos } \\
\text { No. }(\%)\end{array}$ & $\begin{array}{c}\text { Negativos } \\
\text { No. }(\%)\end{array}$ & $\begin{array}{c}\mathrm{RMC}^{\mathrm{C}} \\
(\mathrm{IC} 95 \%)\end{array}$ \\
\hline Mujeres normales & 73 & $1(1 \%)$ & $16(21 \%)$ & $1(1 \%)$ & $55(75 \%)$ & 1.00 \\
\hline Lesión no N Pd & 11 & $2(18 \%)$ & $0 \quad(0 \%)$ & $0(0 \%)$ & $9(82 \%)$ & $0.63(0.1-3.2)$ \\
\hline NIC I y II & 12 & $2(16 \%)$ & $4(33 \%)$ & $2(17 \%)$ & $4(33 \%)$ & $3.9 \quad(1.1-14.04)$ \\
\hline N IC III & 20 & $4(20 \%)$ & $4(20 \%)$ & $0(0 \%)$ & $12(60 \%)$ & $1.9 \quad(0.7-5.3)$ \\
\hline Total & 116 & $9(8 \%)$ & $24(21 \%)$ & $3(3 \%)$ & $80(69 \%)$ & $3.2(1.35-7.5)$ \\
\hline
\end{tabular}

VPH tipificados en las muestras: (a) oncogénicos = tipos 18, 31, 33, 35, 39, 45, 51, 56 y MM9

(b) no oncogénicos = tipos 6, 11 y MM4

(c) RM, razón de momios cruda

(d) N P, neoplásica 


\section{Cuadro II \\ Detección de anticuerpos anti-Ras y anti-E4 y E7 del VPH16 en suero de mujeres CON Lesiones del CÉRVIX UteRINo. MéXICO, 1999-2000}

\begin{tabular}{|c|c|c|c|c|c|c|c|c|c|c|}
\hline \multirow[b]{2}{*}{$\begin{array}{l}\text { Parámetro } \\
\text { n total=116 }\end{array}$} & & \multicolumn{3}{|c|}{ Anticuerpos anti-E7 } & \multicolumn{3}{|c|}{ Anticuerpos anti-E4 } & \multicolumn{3}{|c|}{ Anticuerpos anti-Ras } \\
\hline & & $\begin{array}{c}\text { Prevalencia } \\
n=49(\%)\end{array}$ & $\begin{array}{c}\text { RM* } \\
\text { (IC 95\%) }\end{array}$ & $p$ & $\begin{array}{l}\text { Prevalencia } \\
n=56(\%)\end{array}$ & $\begin{array}{c}\mathrm{RM}^{*} \\
\text { (IC 95\%) }\end{array}$ & $p$ & $\begin{array}{l}\text { Prevalencia } \\
n=40(\%)\end{array}$ & $\begin{array}{c}\mathrm{RM}^{*} \\
\text { (IC 95\%) }\end{array}$ & $p$ \\
\hline Diagnóstico & No. & & & & & & & & & \\
\hline Mujeres normales & (73) & $23(47 \%)$ & 1.00 & & $32(57 \%)$ & 1.00 & & $18(45 \%)$ & 1.00 & \\
\hline \multicolumn{11}{|l|}{ Mujeres con lesión } \\
\hline Lesión no N Pł & (11) & $6(12 \%)$ & - & - & $7(13 \%)$ & - & - & $6(15 \%)$ & - & - \\
\hline N IC I-II & (12) & $6(12 \%)$ & $0.49(0.06-3.7)$ & 0.49 & $7(13 \%)$ & $0.57(0.08-4.4)$ & 0.56 & $8(20 \%)$ & $2.55(0.34-19.2)$ & 0.36 \\
\hline NIC III & (20) & $14(29 \%)$ & $1.93(0.35-10.8)$ & 0.45 & $10(18 \%)$ & $0.41(0.07-2.4)$ & 0.32 & $8(20 \%)$ & $0.59(0.12-3.0)$ & 0.53 \\
\hline $\mathrm{n}$ total $=43$ & & $n=26(60 \%)$ & & & $n=24(56 \%)$ & & & $n=22(51 \%)$ & & \\
\hline \multicolumn{11}{|l|}{ Mujeres VPH(t) } \\
\hline Mujeres normales & (19) & $8(22 \%)$ & 1.00 & & $9(24 \%)$ & 1.00 & & $7(19 \%)$ & 1.00 & \\
\hline Lesión no N P & (2) & $2(6 \%)$ & - & - & $1(3 \%)$ & $1.11(0.06-20.4)$ & 0.94 & $1(3 \%)$ & $1.71(0.09-31.9)$ & 0.71 \\
\hline NIC I-II & (8) & $4(11 \%)$ & $1.37(0.26-7.2)$ & 0.70 & $6(17 \%)$ & $3.33(0.53-20.9)$ & 0.19 & $6(17 \%)$ & $5.14(0.80-32.7)$ & 0.08 \\
\hline NIC III & (8) & $7(19 \%)$ & $9.62(1.0-94.5)$ & 0.05 & $5(14 \%)$ & $1.85(0.34-10.1)$ & 0.47 & $4(11 \%)$ & $1.71(0.32-9.1)$ & 0.52 \\
\hline $\mathrm{n}$ total de lesiones= & & $n=13(72 \%)$ & & & $n=12(67 \%)$ & & & $=11(61 \%)$ & & \\
\hline
\end{tabular}

Edad

\begin{tabular}{lrrlrllllll}
$<30$ años & $(38)$ & $16(32 \%)$ & 1.00 & & $16(28 \%)$ & 1.00 & & $12(30 \%)$ & 1.00 & \\
\hline $31-35$ años & $(20)$ & $9(18 \%)$ & $1.26(0.35-4.5)$ & 0.71 & $11(20 \%)$ & $1.78(0.53-6.0)$ & 0.35 & $7(18 \%)$ & $1.25(0.34-4.6)$ & 0.73 \\
\hline 36 -40 años & $(31)$ & $13(27 \%)$ & $1.04(0.34-3.2)$ & 0.93 & $14(25 \%)$ & $1.19(0.41-3.5)$ & 0.74 & $9(23 \%)$ & $0.89(0.27-2.9)$ & 0.85 \\
\hline $41-45$ años & $(19)$ & $7(14 \%)$ & $0.77(0.21-2.8)$ & 0.70 & $11(20 \%)$ & $2.48(0.71-8.6)$ & 0.17 & $7(18 \%)$ & $1.44(0.38-5.5)$ & 0.62 \\
\hline$>46$ años & $(8)$ & $4(8 \%)$ & $1.14(0.21-6.3)$ & 0.87 & $4(7 \%)$ & $2.41(0.48-12.2)$ & 0.28 & $5(13 \%)$ & $5.55(1.0-31.8)$ & 0.05
\end{tabular}

N o. parejas

\begin{tabular}{|c|c|c|c|c|c|c|c|c|}
\hline 1 a 2 & (97) & 32 (65\%) & 1.00 & & $41(73 \%)$ & 1.00 & $32(80 \%)$ & 1.00 \\
\hline 3 y más & (19) & $17(35 \%)$ & $0.25(0.76-1.1)$ & 0.06 & $15(27 \%)$ & $8.78(2.04-37.7) \quad 0.003$ & $8(20 \%)$ & $1.63(0.47-5.7)$ \\
\hline
\end{tabular}

Fumar

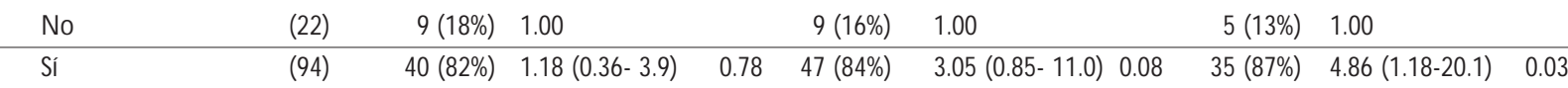

Infección por VPH

\begin{tabular}{|c|c|c|c|c|c|c|c|c|c|c|}
\hline ControlesVPH (-) & (54) & $15(31 \%)$ & 1.00 & & $23(41 \%)$ & 1.00 & & $11(28 \%)$ & 1.00 & \\
\hline ControlesVPH(+) & (19) & $8(16 \%)$ & $2.24(0.7-7.1)$ & 0.17 & $9(16 \%)$ & $1.21(0.38-3.9)$ & 0.74 & $7(18 \%)$ & $2.39(0.69-8.3)$ & \\
\hline CasosVPH (-) & (25) & $13(27 \%)$ & $3.09(0.7-13.3)$ & 0.13 & $12(21 \%)$ & $1.75(0.39-7.9)$ & 0.47 & $12(30 \%)$ & 4.79 (1.11- 20.7) & \\
\hline CasosVPH $(+)$ & (18) & $13(27 \%)$ & $17.80(2.0-157.1$ & 0.01 & $12(21 \%)$ & $5.89(0.85-41.6)$ & 0.07 & $10(25 \%)$ & $6.80(1.08-42.7)$ & \\
\hline
\end{tabular}

* RM : Razón de momios ajustadas por grado de lesión, número de parejas sexuales, fumar e infección por el VPH

₹ N P: N eoplásica

cuerpos anti-E7, en las lesiones NIC III, la cual tuvo una clara tendencia a disminuir con el grado de lesión ( $50 \%$ en NIC I-II y 54\% en lesiones no neoplásicas) lo cual fue estadísticamente significativo $(p=0.009)$. En relación con anticuerpos anti-E4, la prevalencia fue de hasta $64 \%$ (7/11) en lesiones no neoplásicas, y mostró una tendencia inversa al grado de lesión, observándose una prevalencia de $58 \%(7 / 12)$ para las lesiones tipo NIC I-II y de 50\% para las lesiones NIC III (10/20), la misma tendencia fue observada en nuestro laborato- 
rio en un estudio previo, aunque esto no llegó a ser estadísticamente significativo. ${ }^{17}$ Los anticuerpos contra Ras presentaron prevalencias muy altas para las lesiones no neoplásicas $(55 \%, 6 / 11)$ y NIC I-II $(67 \%$, $8 / 12)$, pero se redujo para las lesiones tipo NIC III ( $40 \%$, $8 / 20$ ) siendo esto altamente significativo $(p=0.007)$. Por otra parte, al hacer un análisis por grupo de edad se observó que las prevalencias de anticuerpos anti-Ras fuerón mayores en el grupo de mujeres de más de 46 años de edad $(63 \%, 5 / 8)$, mientras que los demás grupos de edad presentaron prevalencias muy semejantes (de entre 29 a 37\%).

En el grupo con diagnóstico de citología normal se identificó que aproximadamente 34\% (25/73) no presentaron anticuerpos contra ninguna de las proteínas recombinantes utilizadas en el ensayo de ELISA de captura. De este grupo control tenemos que la prevalencia para anticuerpos contra E7 fue de $32 \%$ (23/73), de 44\% (32/73) para anticuerpos anti-E4 y de $25 \%(18 / 73)$ para anticuerpos anti-Ras. Al hacer el análisis final del estudio se identificó que 18 mujeres de este grupo control fueron positivas para algún tipo del VPH, por lo que las prevalencias pudieran estar sobrestimadas.

\section{A sociación de anticuerpos anti-E7, E4 y Ras a factores de riesgo para $\mathrm{CaCu}$}

Teniendo toda la información de los cuestionarios para factores de riesgo y los resultados de laboratorio, se llevó a cabo el análisis estadístico bivariado y multivariado. En el cuadro II se resumen los resultados ajustados para las variables de edad, el grado de lesión, el número de parejas sexuales, el fumar y la presencia de secuencias de DNA del VPH, variables que se asociaron a la presencia de anticuerpos contra las proteínas del VPH y Ras.

En el análisis multivariado la presencia de anticuerpos anti-E7 se correlacionó con diferentes parámetros, pero sólo se encontró una asociación estadísticamente significativa con las mujeres positivas para DNA del VPH que presentaron lesiones NIC III (RM=9.62; IC 95\% 1.0-94.5 p=0.05), asociación que se vio potenciada cuando se consideraron todos los casos positivos para DNA del VPH (RM=17.8; IC 95\% 2.0-157.1; $p=0.01$ ) tomando como referencia a mujeres sin lesión y negativas para el VPH. Es posible que la potencia de este factor se vea incrementado en mayor medida con los casos de cáncer, no incluidos en este estudio.

En el caso de anticuerpos anti-E4 se observó una asociación importante con el número de parejas se- xuales, encontrándose que tener tres o más parejas incrementa en 8.78 veces el riesgo de presentar estos anticuerpos (IC 95\% 2.04- 37.7; $p=0.003$ ). De la misma manera, las mujeres que reportaron haber fumado en algún periodo de su vida presentaron 3.05 veces más riesgo (IC 95\% 0.85-11.0; $p=0.08$ ) de presentar anticuerpos anti-E4, siendo este resultado marginalmente significativo. También se encontró que en los grupos de mujeres de 41 a 45 años de edad y mayores de 46 se incrementó hasta en 2.4 veces el riesgo de desarrollar anticuerpos anti-E4 (IC 95\% 0.71-8.6 y 0.48-12.2, respectivamente), aunque esto no fue estadísticamente significativo, posiblemente porque el número de mujeres por grupo fue reducido.

En relación con la presencia de anticuerpos antiRas se observó que el grupo de mujeres mayores de 46 años de edad tenían 5.5 veces más riesgo de presentar estos anticuerpos (IC 95\% 1.0-31.8), en comparación con las menores de 30 años ( $p=0.05)$. Por otra parte, se observó que el fumar es un factor de riesgo importante para la presencia de este tipo de anticuerpos, y se encontró una asociación de hasta 4.86 veces más alta en las mujeres que fuman comparado con las no fumadoras (IC 95\% 1.18- 20.1; $p=0.03$ ). En cuanto a la presencia de secuencias de DNA del VPH, los anticuerpos anti-Ras se asociaron 4.79 veces más con los casos VPH negativos (IC 95\% 1.11- 20.7, $p=0.04$ ), y hasta 6.8 veces más con los casos VPH positivos (IC 95\% 1.08-42.7; $p=0.04$ ), siendo estos datos estadísticamente significativos. El análisis multivariado también mostró una asociación importante entre anticuerpos anti-Ras y las mujeres con lesiones NIC I-II positivas para DNA de VPH (RM=5.1; IC 95\% 0.8-32-8, $p=0.08)$, lo cual fue marginalmente significativo. Sin embargo, en el análisis bivariado el poder de este factor es más fuerte, ya que el riesgo de desarrollar anticuerpos anti-Ras en las lesiones NIC I-II fue de hasta 6.1 veces, siendo esto altamente significativo (IC 95\% 1.6-22.7; $p=0.007$ ) (datos no mostrados), lo que sugiere que esta asociación es real y su significancia está limitada por el número de observaciones en este grupo.

\section{Perfil de anticuerpos presentes en las mujeres con lesiones del cérvix uterino}

Al tratar de obtener mayor información sobre la presencia de anticuerpos en los sueros de mujeres con diferentes grados de lesión, se evaluó el perfil de anticuerpos que presentaron éstas en comparación con aquellas con citología normal. En el cuadro III se puede observar que más de $93 \%$ (25/27) de las mujeres que fueron negativas para la presencia de cualquiera 


\section{Cuadro III \\ Prevalencia de los diferentes anticuerpos (anti-E4, anti-E7 y anti-Ras) en mujeres CON CITOLOGía NORMAL Y CON DIFERENTES GRAdOS DE LESIÓN DEL CÉRVIX UTERINO. MÉXICO, 1999-2000}

\begin{tabular}{|c|c|c|c|c|c|c|c|c|}
\hline \multirow[b]{2}{*}{ Tipo de lesión } & \multirow[b]{2}{*}{ No. total } & \multicolumn{7}{|c|}{ No. de mujeres positivas para anticuerpos } \\
\hline & & $\begin{array}{c}\text { Negativos* } \\
\text { n (\%) }\end{array}$ & $\begin{array}{c}1 \text { Anticuerpo } \\
n(\%)\end{array}$ & $\begin{array}{c}\mathrm{RM} \\
(\mathrm{IC} 95 \%)\end{array}$ & $\begin{array}{c}2 \text { Anticuerpos } \\
n(\%)\end{array}$ & $\begin{array}{c}\mathrm{RM} \\
\text { (IC 95\%) }\end{array}$ & $\begin{array}{c}3 \text { Anticuerpos } \\
n(\%)\end{array}$ & $\begin{array}{c}\text { RM } \\
\text { (IC 95\%) }\end{array}$ \\
\hline Normal & 73 & $25(34 \%)$ & $27(37 \%)$ & 1.00 & $17(23 \%)$ & 1.00 & $4(5 \%)$ & 1.00 \\
\hline Lesión no N Pł & 11 & $0(0 \%)$ & $2(18 \%)$ & $0.25(.05-1.3)$ & $7(64 \%)$ & $7.4(1.9-28.7)$ & $2(18 \%)$ & $1.8(0.5-9.8)$ \\
\hline NIC I-II & 12 & $1(8 \%)$ & $2(17 \%)$ & $0.22(.04-1.1)$ & $6(50 \%)$ & $4.3(1.2-16.3)$ & $3(25 \%)$ & $2.7(0.6-12.1)$ \\
\hline \multirow[t]{2}{*}{ NIC III } & 20 & $1(5 \%)$ & $7(35 \%)$ & $0.62(0.2-1.7)$ & $6(30 \%)$ & $1.8(0.5-5.6)$ & $6(30 \%)$ & $3.5(1.0-11.6)$ \\
\hline & & & \multicolumn{2}{|c|}{$p$ lineal $=0.14$} & \multicolumn{2}{|c|}{$p$ lineal $=0.08$} & \multicolumn{2}{|c|}{$p$ lineal $=0.03$} \\
\hline Total & 116 & $27(23 \%)$ & \multicolumn{2}{|l|}{$38(33 \%)$} & \multicolumn{2}{|l|}{$36(31 \%)$} & \multicolumn{2}{|l|}{$15(13 \%)$} \\
\hline
\end{tabular}

de los anticuerpos probados fueron del grupo control y menos de $7 \%(2 / 27)$ fueron mujeres con lesiones de tipo NIC I-II y NIC III. En el grupo de sueros de mujeres positivas para un anticuerpo (cualquiera de los tres probados), se observó que $71 \%$ (27/38) de este grupo fueron mujeres normales, mientras que el grupo de aquellas mujeres con algún tipo de lesión se incrementó hasta $29 \%(11 / 38)$ de positivas. Cuando agrupamos a quienes fueron positivas para la presencia de dos anticuerpos la prevalencia se incrementó a 53\% (19/36) en mujeres con lesiones en cérvix, mientras que el grupo control presentó menos de $47 \%$ de positivas para éstos. Finalmente, el grupo de las que dieron positivas para tres de los anticuerpos probados, en su mayor parte se trató del grupo de mujeres con lesiones no neoplásicas, NIC I-II y NIC III (73\%, 11/15), mientras que aquellas con citología normal presentaron una prevalencia de menos de $27 \%$ (4/15). Al realizar el análisis bivariado entre el perfil de anticuerpos y el grado de lesión se encontró que las lesiones no neoplásicas (RM=7.4; IC 95\% 1.9-28.7), y las de tipo NIC I-II (RM 4.3; IC $95 \%$ 1.2-16.3) se asociaron fuertemente con la presencia de dos anticuerpos en suero, siendo esto marginalmente significativo $(p=0.08)$. Sin embargo, la presencia de tres anticuerpos se asoció hasta 3.5 veces más con las lesiones tipo NIC III (IC 95\% 1.0-11.6) y 2.7 veces con las lesiones NIC I-II (IC 95\% 0.6-12.1) lo cual fue estadísticamente significativo $(p=0.03)$. Estos resultados pudieran tener una implicación en un nivel biológico, ya que al realizar el análisis para los sueros que presentaron un sólo anticuerpo, no se encontró una asociación significativa con ninguno de los grupos de mujeres que presentaron algún tipo de lesión.

\section{Discusión}

En este estudio, nos propusimos determinar de manera más precisa el grado de asociación de los anticuerpos anti-E4, E7 y Ras, ya sea de manera individual o en combinación, con las lesiones del tipo NIC I-II y NIC III. Asimismo, se evaluó la utilidad de estos anticuerpos como posibles marcadores tempranos, que permitan aumentar las posibilidades de detectar a mujeres en riesgo de desarrollar $\mathrm{CaCu}$, y que puedan ser tratadas oportunamente.

Hasta ahora la presencia de anticuerpos anti-E4 en el desarrollo del $\mathrm{CaCu}$ no ha sido clara, sin embargo, nuestros resultados mostraron una alta prevalencia para anticuerpos anti-E4 (43\%) en la población control, observándose así un máximo en las lesiones NIC I-II (58\%). Esta prevalencia se vio incrementada hasta en $75 \%$ cuando se consideraron sólo las mujeres VPH positivas, además de correlacionarse de manera importante con la presencia de DNA viral. Estos resultados apoyan lo reportado por Palefsky y colaboradores, ${ }^{30}$ quienes sugieren que la presencia de anticuerpos anti-E4 en los diferentes estadios de la enfermedad se debe a que son producidos tempranamente durante la infección y se mantienen a lo largo del desarrollo del cáncer, lo que podría ser ventajoso para la detección temprana de mujeres con lesiones que pudieran ser de tipo subclínico y progresivas.

Nuestro análisis estadístico también mostró que la seroprevalencia para anticuerpos anti-E7 fue mayor en el grupo de casos con NIC III (70\%), y disminyó a menos de $50 \%$ en los NIC I-II. De igual manera, el grupo de mujeres con NIC III y positivas para el VPH 
fueron hasta en $87 \%$ positivas para anticuerpos antiE7. La asociación de anticuerpos contra proteínas oncogénicas VPH con $\mathrm{CaCu}$ ha sido ampliamente investigada, ${ }^{9,10,31-33}$ sin embargo, nuestros resultados mostraron una alta asociación entre la presencia de anticuerpos anti-E7 y lesiones del tipo NIC III, riesgo que se incrementa hasta en 17.8 veces, si se consideran todos los casos positivos para secuencias del VPH. Esto demuestra que la presencia de anticuerpos anti-E7 está asociada de manera importante con el desarrollo de lesiones NIC III, dato que no había sido reportado en otros estudios y que pudiera ser de utilidad como marcador para este tipo de lesión. Estudios de cohorte han demostrado que las mujeres desarrollan anticuerpos anti-E7 después de varios meses de haber adquirido la infección por el VPH, previo al desarrollo de la lesión de alto grado. ${ }^{34}$ Por lo que la presencia de anticuerpos anti-E7 en la población femenina podría sugerir la aparición de una lesión subclínica, que posiblemente no haya sido detectada por los medios de diagnóstico convencionales.

Por otra parte, se observó una asociación importante entre anticuerpos anti-Ras con los casos, independientemente de la presencia o ausencia de secuencias del VPH, lo cual refleja que los anticuerpos anti-Ras se relacionan con el proceso de transformación y no precisamente con la infección. Se ha sugerido que la presencia de anticuerpos contra proteínas propias del organismo se debe a la pérdida de tolerancia como consecuencia de la acumulación de éstas en las células, o bien a la liberación extracelular de moléculas debida a la presencia de tejido necrótico. Este efecto ha sido descrito para proteínas como p53, Myb y Ras en diferentes tipos de cánceres. ${ }^{35-37}$ La presencia de anticuerpos anti-Ras se ha reportado en pacientes con cáncer de colon, sin haberse encontrado una correlación entre las variables clínicas ni patológicas de los pacientes..$^{35}$ Estudios previos en nuestro laboratorio mostraron una asociación importante entre anticuerpos anti-Ras y lesiones del tipo NIC, aunque en esos trabajos no pudo definirse claramente el estadio (I, II o III) al que se asociaron estos anticuerpos. Hasta ahora no es claro si la respuesta humoral contra Ras pudiera ser de importancia en el desarrollo del cáncer, sin embargo, nuestros resultados mostraron una prevalencia de hasta $67 \%$ con las lesiones del grupo NIC I-II, así como una alta asociación con mujeres $>46$ años de edad, fumadoras $(\mathrm{RM}=4.86)$ y positivas para DNA del VPH. El hecho de que se generen anticuerpos anti-Ras durante la aparición de lesiones tempranas del cérvix uterino, aunado a los otros factores de riesgo, sugiere que éstos podrían ser de utilidad en la detección oportuna de lesiones NIC I-II.
$\mathrm{Al}$ revisar nuestros resultados para identificar el perfil de anticuerpos que presentó la población en estudio, se encontró que mujeres que presentaron dos anticuerpos se asociaron a la presencia de lesiones no neoplásicas y NIC I-II mientras que el tener tres anticuerpos se asoció con las lesiones NIC III, específicamente. Estos resultados sugieren que al investigar la presencia de anticuerpos contra E4, E7 y Ras, el perfil o la combinación de anticuerpos que se encuentren en la mujer, nos podrían estar prediciendo el grado de la lesión que ésta tiene (NIC I, II o III). Con los resultados hasta ahora presentados no es posible definir la secuencia de aparición de los diferentes anticuerpos contra el VPH en relación con el desarrollo de la enfermedad, sin embargo, la alta prevalencia de anticuerpos anti-E4 en la población control sugiere que éstos pudieran estar asociados al desarrollo de una infección productiva, con posibilidades de generar lesiones tempranas en el cérvix uterino. Por lo que el investigar la secuencia de aparición de anticuerpos contra el VPH a diferentes tiempos durante el proceso de desarrollo del cáncer cervical, será de importancia para definir el perfil de anticuerpos contra el VPH, lo cual podría tener un valor predictivo para las neoplasias asociadas a éste.

Las prevalencias para los tres anticuerpos en el grupo control son altas (45-57\%) en relación con lo previamente reportado por nosotros (5-16\%), utilizando Western blot, el cual mostró ser específico para VPH16 $\left(<1 \%\right.$ de reacción cruzada). ${ }^{17}$ Es posible que el incremento en prevalencias se deba a que 18 mujeres del grupo control fueron positivas para algún tipo de VPH $(25 \%)$, prevalencia que fue mayor a lo reportado en la población fuente (14.5\%). ${ }^{26}$ Otra posibilidad es que las prevalencias se hayan incrementado debido a la presencia de reacción cruzada encontrada con los VPH oncogénicos en el ELISA de captura (19\%), porcentaje que es semejante a lo reportado por otros investigadores que utilizaron un ensayo de ELISA convencional $(15 \%) .{ }^{38}$ La posibilidad de haber incurrido en un sesgo de selección del grupo control está presente en nuestro estudio, ya que a pesar de no haber encontrado diferencias estadísticamente significativas en factores de riesgo para $\mathrm{CaCu}$ se observaron diferencias en las prevalencias de infección por el VPH, lo que provocaría una subestimación en la asociación entre la infección por el VPH y la presencia de anticuerpos cuando se compara con la población de casos, es decir, que las asociaciones encontradas podrían ser aún más altas. Por otro lado, el hecho de que exista reacción cruzada con otros VPH oncogénicos pudiera ser de utilidad para poder identificar un mayor número de mujeres infectadas con diferentes VPH, utilizando para ello 
antígenos del VPH16. Los resultados son alentadores, ya que demuestran el potencial de la prueba en la identificación de mujeres infectadas con el VPH en riesgo de desarrollar algún tipo de lesión en el cérvix uterino.

Otro dato importante que se observó en nuestro estudio fue la asociación entre el número de parejas sexuales y el desarrollo del cáncer cervical. Nuestros resultados sugieren que a mayor número de contactos sexuales, mayor será el riesgo de infectarse con algún tipo del VPH y de desarrollar anticuerpos antiE4, lo que se correlaciona directamente con el proceso de infección. Esto tiene sentido en cuanto a que la presencia del virus está supeditada al contacto sexual, lo que origina la infección y, si ésta es persistente, se puede llegar a generar una lesión.

En nuestro estudio también se evaluó la posible influencia del tabaco en el desarrollo del cáncer cervical y la aparición de anticuerpos anti-proteínas tempranas del VPH y anti-Ras. Hasta ahora, no ha sido claro el papel del tabaco en el desarrollo del $\mathrm{CaCu}$, ya que no se ha identificado si éste funciona como factor independiente o como cofactor en la infección por el VPH. Nuestros resultados apoyan la teoría de ser cofactor en el desarrollo de $\mathrm{CaCu}$, ya que pudimos observar que las mujeres que fuman incrementan hasta en 3.05 veces el riesgo de desarrollar anticuerpos anti-E4, y hasta 4.86 veces el presentar anticuerpos anti-Ras, los cuales a su vez se asocian claramente a mujeres con lesiones NIC I-II. De esta manera, nuestros resultados apoyan lo encontrado en los estudios epidemiológicos de Hildesheim ${ }^{39}$ y Moscicki, ${ }^{40}$ quienes sugieren que el fumar es un factor de riesgo importante en la persistencia de la infección por el VPH en lesiones tempranas del cérvix uterino.

En el ELISA de captura que se manejó pudimos apreciar una reacción cruzada con otros tipos del VPH, lo que pudiera estar diluyendo la especificidad de la prueba y la asociación posible, por mala clasificación diagnóstica. Esto debido a que en el grupo control se utilizó el estudio citológico, comparado con el estudio histopatológico para el diagnóstico confirmatorio en los casos, con diferencias en la sensibilidad y especificidad de éstos. Por lo que para valorar la sensibilidad y especificidad del sistema se tomaron 20 muestras de mujeres sin lesión y sin infección por el VPH, de donde se obtuvo una sensibilidad de $67 \%$ y una especificidad de $78 \%$ para esta técnica (datos no mostrados). Es posible que los errores obtenidos en cuanto al valor de corte de cada una de las proteínas provocó una baja sensibilidad, además de que el tamaño de la muestra que se manejó en este trabajo también influyó en ésta. Es sabido que una buena sensibilidad del sistema y un adecuado tamaño de muestra son importantes para que una técnica sea aceptable, ${ }^{29}$ por lo que se espera que utilizando una muestra mayor podamos corregir este error de nuestro sistema.

Finalmente, la posibilidad de utilizar los tres anticuerpos anti-Ras y anti-E4 y E7 de VPH16 juntos, como marcadores en la detección temprana de mujeres en riesgo de desarrollar cáncer cérvico uterino, en combinación con otras técnicas de diagnóstico como el PAP- que permite la identificación de cambios en las células del cérvix asociados a la infección por el VPHy el PCR- empleado para ayudar en la detección de VPH de alto riesgo en mujeres con un PAP positivo, podrían ser muy útiles para discriminar entre una infección por el VPH (VPH positivos por PCR), el grado de la lesión asociada al VPH (presencia de anticuerpos anti-VPH) e incluso determinar aquellas lesiones que puedan persistir o progresar hacia un cáncer (VPH positivos y serología positiva al VPH), siendo esto una herramienta importante en el pronóstico y el consecuente tipo de tratamiento a seguir.

\section{Agradecimientos}

Agradecemos al doctor Kirthi Shah por su valiosa ayuda en la estandarización del sistema de Reverse-line blot en su laboratorio del Departamento de Microbiología Molecular e Inmunología, en Baltimore, MD, EUA. También agradecemos el apoyo técnico, durante la toma de muestras, del grupo de enfermeras de la Clínica de Displasias del Hospital General Doctor Gea González. Igualmente, agradecemos al grupo del doctor Mauricio Hernández Avila por permitirnos trabajar con los bancos de muestras y de datos del estudio poblacional del estado de Morelos, México.

\section{Referencias}

1. zur Hausen H. Papillomavirus infection:A cause of human cancers. Biochim Biophys Acta 1996;1288:F55-F78.

2. Lazcano-Ponce E, Hernández AM, López CL. Factores de riesgo reproductivo e historia de vida sexual asociados a cáncer cervical en una muestra de mujeres de la $\mathrm{C} d$. de México. Rev Invest Clin 1995:47:377-382.

3. Muñoz N . Human papillomavirus and cancer:The epidemiological evidence.J Clin Virol 2000;1:1-5.

4. Farthing AJ,Vousden KH. Functions of human papillomavirus E6 and E7 oncoproteins. Trends Microbiol 1994;2:170-174.

5. Matlashewski G, Schneider J, Banks L, Jones N, Morray A, Crawford L. Human papillomavirus type 16 DN A cooperates with activated ras in transforming primary cells. EMBO J 1987;6:1741-1746.

6. 0 strow RS, Liv Z, Schneider JF, McG lennen RC, Forslund K, Faras AJ. The products of the E5, E6 or E7 open reading frames of RHPV1 can 
individually transform N IH 3T3 cells or in co-transfection with activated ras can transform primary epitelial cells.Virology 1993:196:861-867.

7. D o orbar J, Stherlin J, Mclean L, Crawford L. Specific interaction between HPV-16, E1-E4 and cytokeratins results in collapse of the epithelial cell intermediate filament network. N ature 1991;352:824-827. 8. Doorbar J, Foo CH, Coleman N, Medcalf L, Hartley 0, Prospero T et al. Characterization of events during the late stages of HPV-16 infection in vivo using high-affinity synthetic Fabs to E4.Virol 1997;238:40-52. 9. Jochmus-Kudielka I, Schneider A, Braun R, Kimming R, Koldovsky U, Schneweis KE et al.Antibodies against the human papillomavirus type 16 early proteins in human sera: Correlation of anti-E7 reactivity with cervical cancer. J N a C ancer Inst 1989;81:1698-1704.

10. Fujii T, Matsushima Y, Yajima M, Sugimura T, Terada M. Serum antibody against un-fused recombinant E7 protein of human papillomavirus type 16 in cervical cancer patients. Jpn J Cancer Res 1995;86:28-34. 11.Gosh AK, Smith N K, Stancey SN , G lew SS, Connor ME,Arrand JR et al. Serological responses to HPV-16 in cervical displasia and neoplasia correlation of antibodies to $\mathrm{E} 6$ with cervical cancer. Int J Cancer 1993;53:591-596.

12. Kanda T, 0 ndaT, Z anma S, Yasugi T, Furund $A$, W atanabe $S$ et al. Independent association of antibodies against human papillomavirus type $16 \mathrm{E} 1, \mathrm{E} 4$ and E7 proteins with cervical cancer.Virology 1992;190:724-732.

13. Kirnbauer R, Hubbert LN , Cosette MW, Becker MT, Lowy RD, Schiller TJ.A virus-like particle enzyme-linked immunosorbent assay detects serum antibodies in a majority of women infected with human papillomavirus type 16. J N at Cancer Inst 1994;86:494-498.

14. D oherty PC,A hmed R. Immune responses to viral infection. En: $\mathrm{N}$ atanson $\mathrm{N}$,A hmed R, Ed. Viral Pathogenesis and Immunity. Philadelphia (PA): Lip. Publishers, 1997:143-161.

15. Dillner L, Fredricksson A, Persson E, Forslund O, Hansson BG, Dillner J.Antibodies against papillomavirus antigens in cervical secretions from condyloma patients. J C lin Microbiol 1993;31:192-197. 16. Suchánková A, Krchnak V, Vanger J, H amsikova E, Krcmar M, Ritterova $L$ et al. Epitope mapping of the human papillomavirus type 16 E4 protein by means of synthetic peptides. J G en Virol 1992;73:429-432. 17.Pedroza-SaavedraA, Cruz A, Esquivel F, De LaTorre F, Berumen J, $G$ ariglio $P$ et al. High prevalence of sera antibodies to Ras and to human papillomavirus type $16 \mathrm{E} 4$ proteins in patients with pre-cancerous lesions from the uterine cervix. Arch Virol 2000;145:603-623. 18. Schiffman MH, Brinton LA.The epidemiology of cervical carcinogenesis. Cancer 1995;'75:1988-1901.

19. Riou GF. Proto-oncogenes and prognosis in early carcinoma of uterine cervix. Cancer surveys 1988;7:441-455.

20. Parker MF,Arroyo GF, Geradts J. Molecular characterization of adenocarcinoma of the cervix. Gynecol. 0 ncol 1997;64:242-251. 21.Ishiji T. Molecular mechanism of carcinogenesis by human papillomavirus-16. J Dermatol 2000;27:73-86.

22. G ravitt PE, Peyton CL,Apple RJ,W heeler CM. G enotyping of 27 human papillomavirus types by using L1 consensus PCR products by a single-hybridization reverse line blot detection method.J Clin Microbiol 1998:36:3020- 3027.

23. Hall S, Lorincz A, Shah F, Sherman ME, A bbas F, Paull G et al. Human papillomavirus DNA detection in cervical specimens by hybrid capture: Correlation with cytologic and histologic diagnoses of squamous intraepithelial lesions of the cervix. Gynecol 0 ncol 1996;62:353-359.
24. Muñoz N , Franceschi S, Bosetti C, Moreno V, Herrero R, Smith JS et al. Role of parity and human papillomavirus in cervical cancer:The IARC multicentric case-control study. Lancet 2002;359:1093-1101.

25. Hernández AM, Lazcano PE, Berumen CJ, Cruz VA, Alonso DRP, González LG. Human papilloma virus 16-18 infection and cervical cancer in Mexico:A case-control study. Arch Med Res 1997;28:265-271. 26. Lazcano-Ponce E, Herrero R, Muñoz N, Cruz A, Shah KV,Alonso P et al. Epidemiology of HPV infection among Mexican women with normal cervical cytology. Int J Cancer 2001;91:412-420.

27.Manos MM, Ting Y,W right DK, Lewis AJ, Broker TR, W olinsky SM. The use of polymerase chain reaction amplification for the detection of genital HPV. Cancer Cells 1989;7:209-214.

28.Lautenberger JA, C ourt D, Papas TS. High level expression in E. coli of the carboxy-terminal sequences of the avian myelocytomatosis virus (MC29) v-myc protein. Gene 1983;23:75-84.

29. Meschede W, Zumbach K, Braspenning J, Scheffner M, Benitez-BL, Luande J et al.Antibodies against early proteins of human papillomaviruses as diagnostic markers for invasive cervical cancer. J Clin Microbiol 1998:36:475-480.

30. Palefsky JM, W inkler B, Rabanus JP, C lark C, C han S, N izet V et al . Characterization of in vivo expression of the human papillomavirus type 16 E4 protein in cervical biopsy tissues. J C lin Invest 1991;87(6): 2132-2141.

31.H ashido M, Kanda T, Zanma S, W atanabe S, Komiyama N ,Yoshikawa H et al. Detection of human antibody against the HPV type 16 E7 protein. Jpn J C ancer Res 1991;82:1406-1412.

32. Jha PK, Beral V, Peto J, Hack S, Hermon C, D eacon J et al.Antibodies to human papillomavirus and to other genital infectious agents and invasive cervical cancer risk. Lancet 1993;341:1116-1118.

33. Dillner J, Lenner M, Eklund C, H eino P,W iklund F, Hallmans $G$ et al. A population-based sero-epidemiological study of cervical cancer. $C$ ancer Res 1994;54:134-141.

34. Ho GYF, Bierman R, Beardsley L, Chang Ch], Burk RD. N atural history of cervicovaginal papillomavirus infection in young women. $N$ Eng, J Med 1998;338:423-428.

35.Takahashi M, Chen W, Byrd DR, D isis ML, Huseby ES, Q in H et al. Antibody to ras proteins in patients with colon cancer. Clin Cancer Res 199510:1071-1077.

36. C anevari S, Pupa SM, Ménard S. Revised anti-cancer serological response: Biological significance and clinical implications 1975-1995.Ann Oncol 1996;7:227-232.

37. Soussi T.The humoral response to the tumor-suppressor gene-product p53 in human cancer: implications for diagnosis and therapy. Immunol Today 1996;17:354-356.

38. Hamsikova E, LudvikovaV, Smahel M, Sapp M,Vonka V. Prevalence of antibodies to human papillomaviruses in the general population of the Czech Republic. Int J Cancer 1998;77(5):689-694.

39. Hildesheim A, Herrero R, Castle PE, W acholder S, Bratti MC, Sherman ME, et al. HPV co-factors related to the development of cervical cancer: Results from a population-based study in Costa Rica. $\mathrm{Br}$ J Cancer 2001;84(9):1219-1226.

40. Moscicki AB, Hills N, Shiboski S, Powell K, Jay N , Hanson E et al. Risks for incident human papillomavirus infection and low-grade squamous intraepithelial lesion development in young females. JAMA 2001;285(23):2995-3002. 